\title{
Design of highly focused fields that remain unpolarized on axis
}

\author{
Rosario Martínez-Herrero, ${ }^{1}$ Ignasi Juvells, ${ }^{2}$ and Artur Carnicer ${ }^{2, *}$ \\ ${ }^{1}$ Universidad Complutense de Madrid, Facultad de Ciencias Físicas, Departamento de Óptica, Ciudad Universitaria, \\ 28040 Madrid, Spain \\ ${ }^{2}$ Universitat de Barcelona (UB), Facultat de Física, Departament de Física Aplicada i Òptica, Martí i Franquès 1, \\ 28028 Barcelona, Spain \\ *Corresponding author: artur.carnicer@ub.edu
}

Received July 23, 2014; revised September 12, 2014; accepted September 17, 2014; posted September 22, 2014 (Doc. ID 217633); published October 14, 2014

\begin{abstract}
Research on the properties of highly focused fields mainly involved fully polarized light, whereas partially polarized waves received less attention. The aim of this Letter is to provide an appropriate framework, for designing some features of the focused field, when dealing with incoming partially polarized beams. In particular, in this Letter, we describe how to get an unpolarized field on the axis of a high numerical aperture objective lens. Some numerical results that corroborate theoretical predictions are provided. (C) 2014 Optical Society of America OCIS codes: (260.0260) Physical optics; (260.2110) Electromagnetic optics; (260.5430) Polarization. http://dx.doi.org/10.1364/OL.39.006025
\end{abstract}

The study of field distribution, in the focal region of a high numerical aperture (NA) focusing system, is attracting increasing attention because of their possible applications in many fields, e.g., electron acceleration, nonlinear optics, or particle trapping and manipulation. In general, these techniques require three-dimensional (3D) focused electromagnetic fields, with special characteristics: shape, polarization coherence, and so on. Obtaining these specific features involves the suitable design of the input field [1-6]. Research on the polarization properties of highly focused fields has been mainly devoted on fully polarized light, whereas partially polarized waves have received less attention [7-9]. Moreover, the use of partially coherent fields has been recently proposed as a suitable light source for optical trapping systems [10]; this kind of fields are also useful in tomography [11], plasmonics spectroscopy [12], or invisibility cloaking [13].

Accordingly, the aim of this Letter is to provide an appropriate framework for designing some features of the focused field, when dealing with incoming partially polarized beams. First, we relate the circular components of the transverse incident field with the circular and longitudinal content of the focused field; then, this formalism is extended to quasi-monochromatic statistically stationary incident beams. Finally, we focus on getting an unpolarized field on the axis of an imaging system with a high NA. Moreover, the polarization over the focal plane is also analyzed.

The electric field distribution, at any point in the focal region of a high NA focusing system, is given by the wellknown Richards-Wolf integral [14]

$$
\begin{aligned}
\mathbf{E}(r, \varphi, z)= & A \int_{0}^{\theta_{0}} \int_{0}^{2 \pi} P(\theta) \mathbf{E}_{\mathbf{0}}(\theta, \phi) e^{i k \sin \theta r \cos (\varphi-\phi)} \\
& \times e^{-i k \cos \theta z} \sin \theta \mathrm{d} \theta \mathrm{d} \phi
\end{aligned}
$$

where $A$ is a constant, related to the focal length and the wavelength; $k$ is the wave number, $r$ and $\phi$ denote in this Letter the polar coordinates at the focal plane; and angles $\theta$ and $\theta_{0}$ are represented in Fig. $1 . P(\theta)$ denotes the so-called apodization function. obtained from energy conservation and geometric considerations; and $\mathbf{E}_{\mathbf{0}}$ is the so-called vector angular spectrum; this angular spectrum is usually written as

$$
\mathbf{E}_{\mathbf{0}}(\theta, \phi)=f_{1}(\theta, \phi) \mathbf{e}_{\mathbf{1}}(\phi)+f_{2}(\theta, \phi) \mathbf{e}_{\mathbf{2}}(\theta, \phi),
$$

where $f_{1}$ and $f_{2}$ are, respectively, the azimuthal and radial components of incident field (which we assume transversal). The unitary vectors $\mathbf{e}_{1}$ and $\mathbf{e}_{2}$ are given by

$$
\begin{gathered}
\mathbf{e}_{1}(\phi)=(-\sin \phi, \cos \phi, 0), \\
\mathbf{e}_{2}(\theta, \phi)=(\cos \theta \cos \phi, \cos \theta \sin \phi, \sin \theta) .
\end{gathered}
$$

Frequently, it is useful to write the angular spectrum using a convenient change of basis [15-17]. Instead of using the conventional radial and azimuthal description, it is advisable to describe the angular spectrum in terms of the circular content of the beam (see below). Accordingly, we write the angular spectrum using an alternative form

$$
\mathbf{E}_{\mathbf{0}}(\theta, \phi)=g_{1}(\theta, \phi) \mathbf{v}_{\mathbf{1}}(\theta, \phi)+g_{2}(\theta, \phi) \mathbf{v}_{\mathbf{2}}(\theta, \phi),
$$

where $g_{1}$ and $g_{2}$ are, respectively, the right-hand and lefthand circular components of the incident field; $g_{1}$ and $g_{2}$

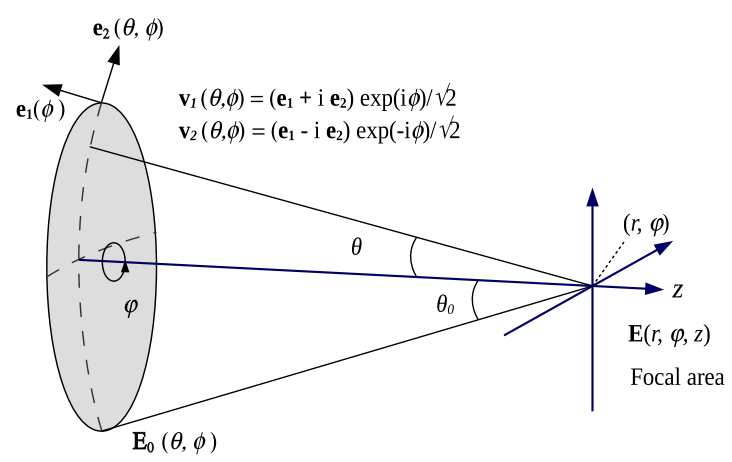

Fig. 1. Geometry and variables involved. 
are related with the radial and azimuthal components, by means of

$$
\begin{aligned}
& g_{1}=\frac{1}{\sqrt{2}}\left(f_{2}-i f_{1}\right) e^{-i \phi}, \\
& g_{2}=\frac{1}{\sqrt{2}}\left(f_{2}+i f_{1}\right) e^{i \phi},
\end{aligned}
$$

where $\mathbf{v}_{\mathbf{1}}$ and $\mathbf{v}_{\mathbf{2}}$ are mutually orthogonal unitary vectors, introduced in [ $\underline{15}-\underline{17}]$

$$
\begin{aligned}
& \mathbf{v}_{\mathbf{1}}(\theta, \phi)=\frac{e^{i \phi}}{\sqrt{2}}\left(\mathbf{e}_{2}+i \mathbf{e}_{1}\right), \\
& \mathbf{v}_{\mathbf{2}}(\theta, \phi)=\frac{e^{-i \phi}}{\sqrt{2}}\left(\mathbf{e}_{2}-i \mathbf{e}_{1}\right) .
\end{aligned}
$$

Note that $\mathbf{v}_{\mathbf{m}}(\theta, \phi) \exp (i \mathrm{kr} \cdot \mathbf{s})$ with $m=1,2$ represents circularly polarized planar waves, propagating along the direction defined by $\mathbf{s}=(\sin \theta \cos \phi, \sin \theta \sin \phi$, $-\cos \theta$ ). Thus, Eq. (4) can be understood as a superposition of right and left circularly polarized plane waves. In particular, in the paraxial limit $(\theta \rightarrow 0), \mathbf{v}_{\mathbf{1}}=\frac{1}{\sqrt{2}}(1, i, 0)$ and $\mathbf{v}_{\mathbf{2}}=\frac{1}{\sqrt{2}}(1,-i, 0)$, are the usual transversal circular plane waves.

We rewrite the field at the vicinity of the focus plane $\mathbf{E}$ as follows

$$
\mathbf{E}=\mathbf{E}_{\mathbf{c}} \hat{U},
$$

where $\hat{U}$ is the unitary matrix

$$
\hat{U}=\left(\begin{array}{ccc}
\frac{1}{\sqrt{2}} & \frac{i}{\sqrt{2}} & 0 \\
\frac{1}{\sqrt{2}} & \frac{-i}{\sqrt{2}} & 0 \\
0 & 0 & 1
\end{array}\right) .
$$

and $\mathbf{E}_{\mathrm{c}}=\left(E_{+}, E_{-}, E_{z}\right)$; in this Letter, $E_{+}$and $E_{-}$represent the right and left circular content of the transverse field, at the vicinity of the focus plane; and $E_{z}$ is the magnitude of the longitudinal component. Taking into account Eqs. (4) and (7), Eq. (1) is rewritten as

$$
\begin{aligned}
\mathbf{E}_{\mathbf{c}}(r, \varphi, z)= & A \int_{0}^{\theta_{0}} \int_{0}^{2 \pi} P(\theta) \mathbf{g}(\theta, \phi) \hat{A}(\theta, \phi) e^{i k \sin \theta r \cos (\phi-\varphi)} \\
& \times e^{-i k \cos \theta z} \sin \theta \mathrm{d} \theta \mathrm{d} \phi
\end{aligned}
$$

where $\mathbf{g}=\left(g_{1}, g_{2}\right)$ and $\hat{A}(\theta, \phi)$ is a $2 \times 3$ matrix given by $\hat{A}(\theta, \phi)=\left(\begin{array}{ccc}\cos ^{2}(\theta / 2) & -\sin ^{2}(\theta / 2) e^{2 i \phi} & \frac{1}{\sqrt{2}} \sin \theta e^{i \phi} \\ -\sin ^{2}(\theta / 2) e^{-2 i \phi} & \cos ^{2}(\theta / 2) & \frac{1}{\sqrt{2}} \sin \theta e^{-i \phi}\end{array}\right)$.

Thus, Eq. (9) relates the circular components of the incident field, with the circular and longitudinal content of the focused field. It should be noted that incident right (left) polarized beams (i.e., incident beams with $g_{1}$ or $g_{2}$ equal to zero) produce, at the focal plane, fields whose transverse component has elliptic polarization. Moreover, according to Eq. (9), a focused field whose transverse component is circularly polarized at any plane $z$ is obtained, if and only if the incident beam satisfies

$$
g_{1}(\theta, \phi)=g_{2}(\theta, \phi)(\tan \theta / 2)^{2} e^{-2 i \phi},
$$

or

$$
g_{2}(\theta, \phi)=g_{1}(\theta, \phi)(\tan \theta / 2)^{2} e^{2 i \phi} .
$$

Note that recently, an experimental implementation of such beams has been reported [18]. Let us next consider a quasi-monochromatic statistically stationary incident beam characterized by means of the $2 \times 2$ matrix $\hat{G}$, defined as

$$
\hat{G}\left(\theta_{1}, \phi_{1}, \theta_{2}, \phi_{2}\right)=\left\langle\mathbf{g}^{\dagger}\left(\theta_{1}, \phi_{1}\right) \mathbf{g}\left(\theta_{2}, \phi_{2}\right)\right\rangle,
$$

where the dagger $\dagger$ stands for transpose complex conjugate; and the angular brackets indicate statistical average. Matrix $\hat{G}$ is related with the $2 \times 2$ cross spectral density matrix $\hat{W}_{0}$ of the incident beam [19] by means of

$$
\hat{G}=U_{0} \hat{W}_{0} U_{0}^{\dagger},
$$

with

$$
\hat{U}_{0}=\frac{1}{\sqrt{2}}\left(\begin{array}{cc}
1 & i \\
1 & -i
\end{array}\right)
$$

Therefore, $\hat{G}$ contains the coherence-polarization features of the input paraxial beam. Taking into account Eq. (7), the coherence-polarization properties of the focused field are fully contained in the $3 \times 3$ cross-spectral density matrix $\hat{W}_{c}$ given by

$$
\hat{W}_{c}\left(r_{1}, \varphi_{1}, r_{2}, \varphi_{2}, z\right)=\left\langle\mathbf{E}_{\mathbf{c}}^{\dagger}\left(r_{1}, \varphi_{1}, z\right) \mathbf{E}_{\mathbf{c}}\left(r_{2}, \varphi_{2}, z\right)\right\rangle .
$$

From Eqs. (9), (13), and (15), it follows that $\hat{W}_{c}$ and $\hat{G}$ are related, by means of

$$
\begin{aligned}
& \hat{W}_{c}\left(r_{1}, \varphi_{1}, r_{2}, \varphi_{2}, z\right) \\
& =|A|^{2} \int_{0}^{\theta_{0}} \int_{0}^{\theta_{0}} \int_{0}^{2 \pi} \int_{0}^{2 \pi} \hat{A}\left(\theta_{1}, \phi_{1}\right)^{\dagger} \hat{G}\left(\theta_{1}, \phi_{1}, \theta_{2}, \phi_{2}\right) \hat{A}\left(\theta_{2}, \phi_{2}\right) \\
& \quad \times P\left(\theta_{1}\right) P\left(\theta_{2}\right) e^{-i k \mathbf{r}_{1} \cdot \mathbf{s}_{1}} e^{i k_{1} \mathbf{r}_{2} \cdot \mathbf{s}_{2}} \sin \theta_{1} \sin \theta_{2} \mathrm{~d} \theta_{1} \mathrm{~d} \theta_{2} \mathrm{~d} \phi_{1} \mathrm{~d} \phi_{2} .
\end{aligned}
$$

Note that $\mathbf{r}_{j}=\left(r_{j} \cos \phi_{j}, r_{j} \sin \phi_{j}, z\right)$ and $\mathbf{s}_{j}=\left(\sin \theta_{j}\right.$ $\cos \varphi_{j}, \sin \theta_{j} \sin \varphi_{j},-\cos _{j} \theta_{j}$ ) with $j=1,2$. Equation (17) is one of the main results of this Letter. This formula provides the relationship between the properties of coherence-polarization of the incident beam. and the focused field, in terms of the circular polarization content. It appears especially suitable for designing some predetermined features of the field at the vicinity of the focal region.

Next, we turn our attention to obtain a nonpolarized field on the axis. Taking Eq. (17) into account, the cross spectral density matrix on axis can be written as 


$$
\begin{aligned}
\hat{W}_{c}(0, z)= & |A|^{2} \int_{0}^{\theta_{0}} \int_{0}^{\theta_{0}} \int_{0}^{2 \pi} \int_{0}^{2 \pi} \hat{A}\left(\theta_{1}, \phi_{1}\right)^{\dagger} \hat{G}\left(\theta_{1}, \phi_{1}, \theta_{2}, \phi_{2}\right) \\
& \times \hat{A}\left(\theta_{2}, \phi_{2}\right) P\left(\theta_{1}\right) P\left(\theta_{2}\right) e^{-i k z \cos \theta_{2}} e^{i k z \cos \theta_{1}} \sin \theta_{1} \\
& \times \sin \theta_{2} \mathrm{~d} \theta_{1} \mathrm{~d} \theta_{2} \mathrm{~d} \phi_{1} \mathrm{~d} \phi_{2} .
\end{aligned}
$$

Then, in order to have a nonpolarized field on axis, it is sufficient that $\hat{W}_{c}(0, z)$ takes the form

$$
\hat{W}_{c}(0, z)=J(0, z) \hat{I}_{3},
$$

where $\hat{I_{3}}$ is the $3 \times 3$ identity matrix; and $J(0, z)$ is any positive function. It is important to stress that for a nonpolarized incident beam, the field on axis is partially polarized.

Let us consider an incident beam, whose cross spectral density matrix $\hat{G}$ is given by

$$
\hat{G}\left(\theta_{1}, \phi_{1}, \theta_{2}, \phi_{2}\right)=\hat{B}^{\dagger}\left(\theta_{1}, \phi_{1}\right) \hat{G}_{0}\left(\theta_{1}, \phi_{1}, \theta_{2}, \phi_{2}\right) \hat{B}\left(\theta_{2}, \phi_{2}\right),
$$

being

$$
\begin{aligned}
\hat{G_{0}}\left(\theta_{1}, \phi_{1}, \theta_{2}, \phi_{2}\right)= & g\left(\theta_{1}, \theta_{2}\right)\left(2 \sin \theta_{1} \sin \theta_{2} \hat{I}_{2}\right. \\
& \left.+\cos \theta_{1} \cos \theta_{2} \mathbf{F}\left(\phi_{1}\right)^{\dagger} \mathbf{F}\left(\phi_{2}\right)\right) .
\end{aligned}
$$

In this Letter, $g\left(\theta_{1}, \theta_{2}\right)$ is a nonnegative definite function, fulfilling $g\left(\theta_{1}, \theta_{2}\right)^{*}=g\left(\theta_{2}, \theta_{1}\right) ; \hat{I}_{2}$ is the $2 \times 2$ identity matrix; $\mathbf{F}$ is the row vector $\mathbf{F}(\phi)=\left(e^{-i \phi}, e^{i \phi}\right)$; and $\hat{B}$ is the $2 \times 2$ matrix defined as

$$
\hat{B}(\theta, \phi)=\left(\begin{array}{cc}
\cos ^{2}(\theta / 2) & \sin ^{2}(\theta / 2) e^{2 i \phi} \\
\sin ^{2}(\theta / 2) e^{-2 i \phi} & \cos ^{2}(\theta / 2)
\end{array}\right) .
$$

From a physical point of view, $\hat{G}_{0}$ represent the incoherent superposition, with different weights, of both an unpolarized beam and a totally polarized beam; as a result of superposition, the total field is partially polarized nonuniformly. In fact, the conventional two-dimensional (2D)-degree of polarization takes the form

$$
P_{2 d}(\theta)=\frac{\cos ^{2} \theta}{1+\sin ^{2} \theta} .
$$

If this beam passes through an optical element, whose Jones matrix is given by Eq. (22), then the cross spectral density matrix $G$ is given by Eq. (21). To experimentally implement $\hat{G}$, it is important point out that in this Letter, the cross spectral density matrix is handled within the framework of its circular components. Finally, by substituting Eq. (20) into Eq. (17), the elements of matrix $\hat{W}_{c}(r, \varphi, r, \varphi, z)$ are obtained

$$
\begin{aligned}
\hat{W}_{c}(r, \varphi, r, \varphi, z)_{11}= & \hat{W}_{c}(r, \varphi, r, \varphi, z)_{22} \\
= & \int_{0}^{\theta_{0}} \int_{0}^{\theta_{0}} K\left(\theta_{1}, \theta_{2}, z\right) g\left(\theta_{1}, \theta_{2}\right)\left(2 \sin 2 \theta_{1} \sin 2 \theta_{2} J_{0}\left(k r \sin \theta_{1}\right) J_{0}\left(k r \sin \theta_{2}\right)\right. \\
& \left.+4 \cos ^{2} \theta_{1} \cos ^{2} \theta_{2} J_{1}\left(k r \sin \theta_{1}\right) J_{1}\left(k r \sin \theta_{2}\right)\right) \mathrm{d} \theta_{1} \mathrm{~d} \theta_{2}
\end{aligned}
$$

$$
\begin{gathered}
\hat{W}_{c}(r, \varphi, r, \varphi, z)_{12}=\hat{W}_{c}(r, \varphi, r, \varphi, z)_{21}^{*} \\
\left.=4 e^{2 i \varphi} \int_{0}^{\theta_{0}} \int_{0}^{\theta_{0}} K\left(\theta_{1}, \theta_{2}, z\right) g\left(\theta_{1}, \theta_{2}\right) \cos ^{2} \theta_{1} \cos ^{2} \theta_{2} J_{1}\left(k r \sin \theta_{1}\right) J_{1}\left(k r \sin \theta_{2}\right)\right) \mathrm{d} \theta_{1} \mathrm{~d} \theta_{2}, \\
\hat{W}_{c}(r, \varphi, r, \varphi, z)_{13}=\hat{W}_{c}(r, \varphi, r, \varphi, z)_{31}^{*}=2 \sqrt{2} i e^{i \varphi} F(r, z), \\
\hat{W}_{c}(r, \varphi, r, \varphi, z)_{23}=\hat{W}_{c}(r, \varphi, r, \varphi, z)_{32}^{*}=2 \sqrt{2} i e^{-i \varphi} F(r, z), \\
\hat{W}_{c}(r, \varphi, r, \varphi, z)_{33}=\int_{0}^{\theta_{0}} \int_{0}^{\theta_{0}} K\left(\theta_{1}, \theta_{2}, z\right) g\left(\theta_{1}, \theta_{2}\right)\left(8 \sin ^{2} \theta_{1} \sin ^{2} \theta_{2} J_{1}\left(k r \sin \theta_{1}\right) J_{1}\left(k r \sin \theta_{2}\right)\right. \\
\left.+2 \sin 2 \theta_{1} \sin 2 \theta_{2} J_{0}\left(k r \sin \theta_{1}\right) J_{0}\left(k r \sin \theta_{2}\right)\right) \mathrm{d} \theta_{1} \mathrm{~d} \theta_{2},
\end{gathered}
$$

where we denote

and

$$
K\left(\theta_{1}, \theta_{2}, z\right)=\pi^{2}|A|^{2} P\left(\theta_{1}\right) P\left(\theta_{2}\right) e^{-i k z \cos \theta_{2}} e^{i k z \cos \theta_{1}} \sin \theta_{1} \sin \theta_{2},
$$

$$
\begin{aligned}
F(r, \varphi)= & \int_{0}^{\theta_{0}} \int_{0}^{\theta_{0}} K\left(\theta_{1}, \theta_{2}, z\right) g\left(\theta_{1}, \theta_{2}\right)\left(\sin 2 \theta_{1} \sin ^{2} \theta_{2} J_{0}\left(k r \sin \theta_{1}\right) J_{1}\left(k r \sin \theta_{2}\right)\right. \\
& \left.-\sin 2 \theta_{2} \cos ^{2} \theta_{1} J_{1}\left(k r \sin \theta_{1}\right) J_{0}\left(k r \sin \theta_{2}\right) \mathrm{d} \theta_{1} \mathrm{~d} \theta_{2}\right) .
\end{aligned}
$$

Then, on axis we get

$$
\hat{W}_{c}(0, z)=2 \hat{I}_{3} \int_{0}^{\theta_{0}} \int_{0}^{\theta_{0}} K\left(\theta_{1}, \theta_{2}, z\right) g\left(\theta_{1}, \theta_{2}\right) \sin ^{2} \theta_{1} \sin ^{2} \theta_{2} \mathrm{~d} \theta_{1} \mathrm{~d} \theta_{2}
$$




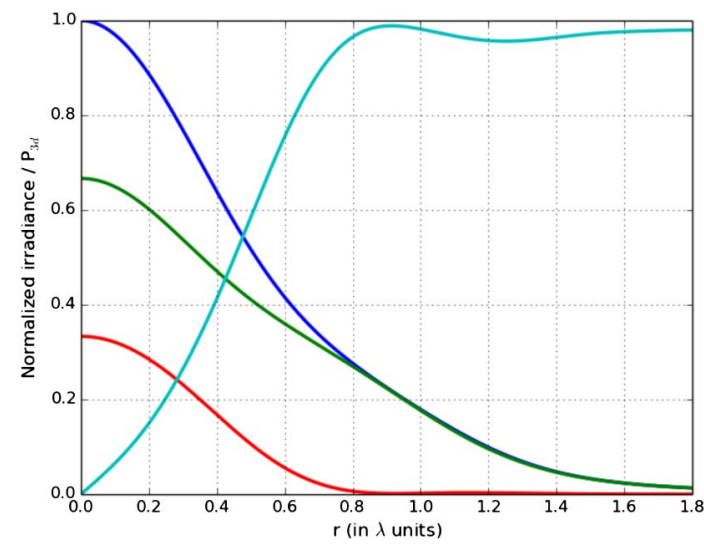

Fig. 2. Irradiance profiles $I_{T}$ (blue), $I_{t}$ (green), $I_{z}$ (red), and 3D-degree of polarization $P_{3 D}$ (turquoise); $\theta_{0}=\pi / 2, f_{0}=0.5$, and $z=0$.

Hence, regardless of the form of function $g$, we always have an unpolarized field on axis. However, out of axis the field exhibits nonuniform partial polarization.

In order to illustrate the behavior of this kind of beams, the 3D-degree of polarization $P_{3 D}$ and the profile of the transversal $\left(I_{t}=W_{11}+W_{22}\right)$, longitudinal $\left(I_{z}=W_{33}\right)$, and total $\left(I_{T}=I_{t}+I_{r}\right)$ irradiances are calculated as a function of $r$. Although several measures for the 3Ddegree of polarization have been proposed (see, for instance, [20] and references therein), in what follows the $P_{3 D}$ is calculated according to Eq. $(32)[3,8]$

$$
P_{3 D}^{2}=\frac{3}{2}\left(\frac{\operatorname{tr}\left(W_{c} W_{c}\right)}{\left(\operatorname{tr} W_{c}\right)^{2}}-\frac{1}{3}\right)
$$

In this way, it is possible to compare the results, presented in Figs. 2 and 3, with those obtained when dealing with other problems that require the calculation of the 3D-degree of polarization (see, for instance, []]).

In the cases analyzed, a factorizable function $g$ is selected

$$
g\left(\theta_{1}, \theta_{2}\right)=g_{0}\left(\theta_{1}\right) g_{0}\left(\theta_{2}\right),
$$

being $g_{0}$ of Gaussian profile, i.e.,

$$
g_{0}(\theta)=\exp \left(-\frac{\sin \theta}{f_{0} \sin \theta_{0}}\right)^{2},
$$

where $f_{0}$ is the so-called filling factor. Figures 2 and 3 show the irradiance profiles and $P_{3 D}$, for $f_{0}=\overline{0} .5$ and $f_{0}=2$, respectively. In Figs. 2 and $3, z=0$ and $\theta_{0}=\pi / 2$.

Regarding the behavior of the $3 \mathrm{D}$-degree of polarization for $f_{0}=0.5, P_{3 D}$ is equal to zero on axis, as expected. Then, its value rises until $P_{3 D} \approx 1$, for $r>0.8 \lambda$. For the case $f_{0}=2, P_{3 D}=0$ at $z=0 . P_{3 D}$ reaches a maximum at $r \approx 1.2 \lambda$, but suddenly drops and again reaches a maximum. Then, an incident partially polarized beam produces a field at the focal region, whose degree of polarization ranges from zero to nearly 1 . This oscillating behavior is in consonance with what is described in [8].

In summary, we developed a theoretical framework for designing partially polarized fields in the focal area. In

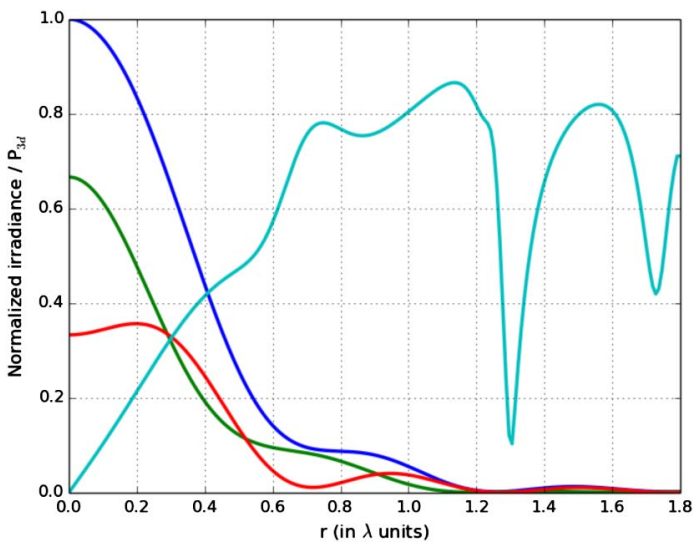

Fig. 3. Irradiance profiles $I_{T}$ (blue), $I_{t}$ (green), and $I_{z}$ (red). 3D-degree of polarization $P_{3 D}$ (turquoise); $\theta_{0}=\pi / 2, f_{0}=2$, and $z=0$.

particular, we centered our attention in a family of beams that remain unpolarized, on the axis of a high NA objective lens. Numerical results corroborate that out of the axis, the field remains partially polarized.

This Letter has been supported by the Ministerio de Ciencia e Innovación of Spain, project FIS2010-17543, and Ministerio de Economía y Competitividad projects FIS2013-46475.

\section{References}

1. Z. Chen and D. Zhao, Opt. Lett. 37, 1286 (2012).

2. C. J. Sheppard and S. Mehta, Opt. Express 20, 27212 (2012).

3. T. Setälä, A. Shevchenko, M. Kaivola, and A. T. Friberg, Phys. Rev. E 66, 016615 (2002).

4. F. Kenny, D. Lara, O. Rodrguez-Herrera, and C. Dainty, Opt. Express 20, 14015 (2012).

5. R. Martnez-Herrero, I. Juvells, and A. Carnicer, Opt. Lett. 38, 2065 (2013).

6. D. Maluenda, I. Juvells, R. Martnez-Herrero, and A. Carnicer, Opt. Express 21, 5432 (2013).

7. K. Lindfors, T. Setälä, M. Kaivola, and A. T. Friberg, J. Opt. Soc. Am. A 22, 561 (2005)

8. K. Lindfors, A. Priimagi, T. Setälä, A. Shevchenko, A. T. Friberg, and M. Kaivola, Nat. Photonics 1, 228 (2007).

9. L.-P. Leppänen, A. T. Friberg, and T. Setälä, J. Opt. Soc. Am. A 31, 1627 (2014).

10. J. M. Auñón and M. Nieto-Vesperinas, Opt. Lett. 38, 2869 (2013).

11. K. H. Kim, B. H. Park, Y. Tu, T. Hasan, B. Lee, J. Li, and J. F. de Boer, Opt. Express 19, 552 (2011).

12. J. A. Fan, K. Bao, J. B. Lassiter, J. Bao, N. J. Halas, P. Nordlander, and F. Capasso, Nano Lett. 12, 2817 (2012).

13. T. Ergin, N. Stenger, P. Brenner, J. B. Pendry, and M. Wegener, Science 328, 337 (2010).

14. B. Richards and E. Wolf, Proc. R. Soc. London, Ser. A 253, 358 (1959).

15. Q. Zhan, Opt. Lett. 31, 867 (2006).

16. R. Martnez-Herrero and P. Mejas, Opt. Express 18, 7965 (2010).

17. A. Y. Bekshaev, Central Eur. J. Phys. 8, 947 (2010).

18. D. Maluenda, R. Martnez-Herrero, I. Juvells, and A. Carnicer, Opt. Express 22, 6859 (2014).

19. E. Wolf, Introduction to the Theory of Coherence and Polarization of Light (Cambridge University, 2007).

20. M. R. Dennis, J. Opt. Soc. Am. A 24, 2065 (2007). 